\title{
Digitale Transformation ganzheitlich managen
}

\author{
Wie Unternehmen die digitale Transformation \\ strukturiert meistern
}

\author{
Roman Dumitrescu, André Lipsmeier, Thorsten Westermann und Arno Kühn, \\ Fraunhofer-Institut für Entwurfstechnik Mechatronik IEM, Paderborn
}

\begin{abstract}
Digitalisierung ist ein Kernthema, das in den Strategien von Unternehmen berücksichtigt werden sollte. Aufgrund der unternehmensspezifischen Ausgangssituationen und Ziele existiert jedoch kein einheitliches Muster für die digitale Transformation. So sollte jedes Unternehmen seine eigene Strategie für die Gestaltung der Digitalisierung entwickeln. Dabei ist zu festzulegen, in welchen Bereichen ein Unternehmen nutzenstiftende Veränderungen im Kontext der Digitalisierung durchführen will.
\end{abstract}

Ob Vertrieb, Rechnungswesen oder Produktion - die Digitalisierung erfordert vielfältige Veränderungen in unterschiedlichen Unternehmensbereichen. Um diese Veränderungen strukturiert zu steuern und nachhaltig umzusetzen, bedarf es einen als „Digitale Transformation" bezeichneten Prozess. Zur Strukturierung der hierfür erforderlichen Aktivitäten nutzen wir vier übergeordnete Handlungsfelder. Das Handlungsfeld Marktleistung beschreibt die Veränderung des Produkt- und Serviceprogramms, bei dem Produkt- und Dienstleistungsanteile zunehmend zu integrierten Produkt-Service-Systemen verschmelzen. Im Handlungsfeld Leistungserstellung wird die Frage beantwortet, wie Geschäftsprozesse und Unternehmensressourcen für eine zukünftige Wertschöpfung verändert werden müssen. Im Fokus des Handlungsfelds Geschäftsmodelle steht die Entwicklung digitaler Geschäftsmodelle, die zu einer nutzenstiftenden Veränderung der Geschäftslogik eines Unternehmens führen. Als Grundlage zur Umsetzung dieser drei Handlungsfelder gilt das vierte Handlungsfeld Organisation. Erforderliche Initiativen für die Anpassung der Aufbauund Ablauforganisation, zur Erzeugung einer strategiekonformen Kultur und letztlich zur Erzeugung einer digitalen Arbeitswelt im Unternehmen, werden dort adressiert.

\section{Digitale Transformation von Unternehmen}

Jedes Unternehmen beginnt seinen Transformationsprozess von einer individuellen Ausgangsposition und verfolgt zugleich unternehmensspezifische Ziele. Ein standardisierter Fahrplan für die digitale Transformation besteht somit nicht [1]. In Strategieprojekten mit Unternehmen der Maschinenbau-, Nutzfahrzeug- und Hausgerätebranche haben wir jedoch ein dreistufiges Vorgehen entwickelt und validiert. Dazu haben wir den grundlegenden Prozess zur Strategieentwicklung in Anlehnung an [2] adaptiert und einzelne Stufen mit neuen Methoden angereichert. Unter Zuhilfenahme dieses dreistufigen Vorgehens können Unternehmen ihre individuelle digitale Transformation strukturiert planen und realisieren (Bild 1).

Zu Beginn des Transformationsprozesses bewerten Unternehmen ihre derzeitige Leistungsfähigkeit im Kontext der Digitalisierung. Davon ausgehend werden im nächsten Schritt konkrete Digitalisierungsziele festgelegt. Anschließend definieren die Unternehmen konkrete Maßnahmen und Aktivitäten zur Erreichung der Ziele und bringen diese im Rahmen einer Roadmap in eine zeitliche Abfolge. Begleitend
Prof. Dr.-Ing. Roman Dumitrescu ist Direktor am Fraunhofer-Institut für Entwurfstechnik Mechatronik IEM in Paderborn und leitet dort den Fachbereich Produktentstehung. An der Universität Paderborn leitet er den Lehrstuhl für Advanced Systems Engineering und ist außerdem einer von drei Geschäftsführern des Spitzenclusters it's OWL.

M. Sc. André Lipsmeier ist wissenschaftlicher Mitarbeiter in der Gruppe "Zukunfsorientierte UnternehmensEntwurfstechnik IEM in Paderborn.

Dr.-Ing. Thorsten Westermann leitet die Gruppe „Produkt-Service-SysEntwurfstechnik Mechatronik IEM in Paderborn

Dr.-Ing. Arno Kühn leitet die Abteilung "Produkt- und Produktionsmanage ment" am Fraunhofer Institut für Entwurfstechnik IEM in Paderborn.

roman.dumitrescu@iem. fraunhofer.de

www.iem.fraunhofer.de 


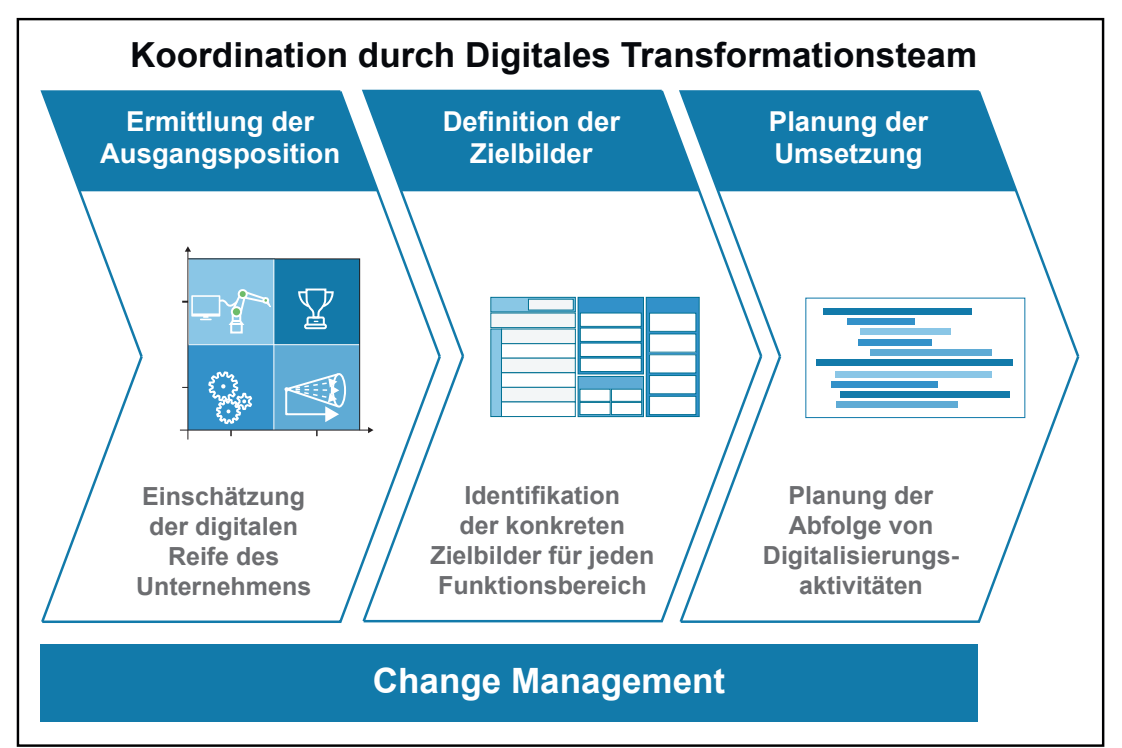

Bild 1: Vorgehensweise zur Planung der digitalen Transformation.

dazu erfolgt das Change Management. Dieses stellt sicher, dass die wesentlichen Stakeholder im Unternehmen bei der Planung und Umsetzung von Digitalisierungsaktivitäten involviert werden. Im Folgenden wollen wir den von uns systematisierten Weg zur Planung der digitalen Transformation von Unternehmen am Beispiel des Handlungsfelds Leistungserstellung erläutern. In den von uns durchgeführten Industrieprojekten haben wir dabei die Erfahrung gemacht, dass Unternehmen vor allem bei der systematischen Bestimmung konkreter Digitalisierungsziele vor Herausforderungen stehen. Nachdem wir das Vorgehen zur Ermittlung der Ausgangssituation beschrieben haben, werden wir deshalb detailliert auf die systematische Zielermittlung durch die Entwicklung sogenannter digitaler Zielbilder eingehen.

\section{Ermittlung der Ausgangssituation}

Ausgangspunkt des digitalen Transformationsprozesses ist eine objektive Bewertung der derzeitigen Leistungsfähigkeit eines Unternehmens im Kontext der Digitalisierung. Empfehlenswert dafür sind Reifegradmodelle, mit deren Hilfe Unternehmen ihren Status Quo systematisch bewerten können. Im Kontext von Digitalisierung und Industrie 4.0 entstanden in jüngerer Vergangenheit mannigfaltige Reifegradmodelle mit verschiedenen Schwerpunkten, z. B. in den Bereichen Produktion oder Logistik [3, 4]. Ein Großteil dieser Modelle greift jedoch zu kurz, da vordergründig technologische Aspekte adressiert werden.

Wie eingangs erläutert, ist die Digitalisierung im Unternehmen jedoch ein mehrdimensionales Thema, bestehend aus technischen sowie strategischen, organisatorischen und kulturellen Aspekten. Vor diesem Hintergrund haben wir ein neues Reifegradmodell entwickelt, das dieser Herausforderung gerecht wird. Es verfolgt eine ganzheitliche Herangehensweise, die auf zwei übergeordneten Dimensionen fußt: „Digital Capabilities" und „Digital Awareness". Die Dimension „Digital Capabilities" repräsentiert technologische sowie infrastrukturelle Fähigkeiten eines Unternehmens im Kontext der Digitalisierung. Die zweite Dimension „Digital Awareness" umfasst strategische, organisatorische und kulturelle Aspekte der Digitalisierung. Diese beiden Dimensionen unterteilen wir jeweils in sechs Handlungsfelder, wie z. B. Datennutzung, IT-Infrastruktur und Ressourcen oder Assistenzsysteme in der Dimension Digital Capabilities (Bild 2). Jedes dieser Handlungsfelder hat unterschiedliche Reifegrade, welche die Eigenschaften eines Handlungsfelds je Leistungsstufe idealtypisch beschreiben. So reichen z.B. die Reifegrade des Kriteritums Prozessunterstützung in der Dimension Digital Capabilites von „Keiner Prozessunterstützung" in der ersten Reifegradstufe bis hin zu "Selbststeuernden Prozessen“ in der vierten Reifegradstufe.

Mithilfe dieses zweidimensionalen Reifegradmodells können sämtliche Funktionsbereiche eines Unternehmens (z. B. Engineering, Produktion, Personalwesen etc.) ihre bereichsspezifische Leistungsfähigkeit in puncto Digitalisierung ermitteln. In Workshops untersuchen Mitarbeiterinnen und Mitarbeiter die Prozesse ihres Funktionsbereichs anhand der Handlungsfelder und bewerten sie mithilfe der einzelnen Kriterien (Bild 3) sowie der zugeordneten Reifegrade. Aus den ermittelten Reifegradstufen wird ein Durchschnittswert für die zwei Dimensionen „Digital Capabilites“ und "Digital Awareness" gebildet, der schließlich in das Ergebnisportfolio einfließt. Das Portfolio in Anlehnung an [5] wird durch die übergeordneten Dimensionen "Digital Capabilities" (y-Achse) und „Digital Awareness" (x-Achse) aufgespannt und dient zur prägnanten Ergebnisdarstellung. Aufgeteilt ist das Ergebnisportfolio in vier verschiedene Bereiche. Der Bereich "Digital Novice" beschreibt Funktionsbereiche mit geringen technischen Fähigkeiten und einer geringen „Digital Awareness". Hier steckt die Digitalisierung noch in den Kinderschuhen. Der Bereich „Technical Pioneer" umfasst Funktionsbereiche mit guten technischen Fähigkeiten bei geringen Werten im Kontext Strategie, Organisation und Kultur. Zwar befinden sich digitale Technologien bereits im operativen Einsatz, jedoch folgt der Technologieeinsatz keiner übergeordneten Strategie. Funktionsbereiche im Feld "Strategic Expert" haben bereits eine klare Strategie für ihren Bereich im Kontext der 
Digitalisierung erstellt und Zuständigkeiten für Digitalisierungsaktivitäten definiert. Es fehlt jedoch noch der operative Einsatz digitaler Technologien. Den Bereich „Digital Champion" erreichen Funktionsbereiche dann, wenn konkrete Maßnahmen zur digitalen Transformation anhand einer klaren Strategie umgesetzt werden. Mit der Einordung der untersuchten Bereiche in das Ergebnisportfolio ist die Analyse der Ausgangssituation abgeschlossen. Nun gilt es den unternehmensindividuellen Weg zum digitalen Champion zu planen und umzusetzen.

\section{Ermittlung von Digitalisierungszielen}

Jedes Unternehmen sollte einen eigenen, unternehmensspezifischen Plan - eine Digitalisierungsstrategie - entwickeln und damit festlegen, wie es sich "digital transformieren" will. Sie beschreibt die gesamtheitliche und spezifische Vision im Kontext der Digitalisierung einschließlich der strategischen Maßnahmen zu deren Erreichung. Dazu definieren Unternehmen konkrete, mittel- sowie langfristige Digitalisierungsziele und -initiativen für die Bereiche Marktleistung und Wertschöpfungsprozesse aber auch für die Bereiche Organisation und Kultur.

Aufgrund der hohen Komplexität der digitalen Transformation empfehlen wir für die Erarbeitung der unternehmensspezifischen Digitalisierungsstrategie einen sogenannten „DownUp"-Ansatz. Dabei wird zunächst ein erster Entwurf einer digitalen Unternehmensvision von der Unternehmensleitung erarbeitet und in Form eines digitalen Leitbilds schriftlich fixiert. Die grundsätzlichen Leitplanken für die Ausgestaltung der digitalen Transformation werden somit von der Unternehmensleitung festgelegt. Weiter konkretisiert werden diese Vorgaben durch die einzelnen Funktionsbereiche des Unternehmens: Hier werden konkrete Digitalisierungsziele und -initiativen erarbeitet, synchronisiert und „Buttom-Up“ in die digitale Unternehmensvision zurückgespielt. Auf der Basis dieser nun konkreten Angaben wird der erste Entwurf der digitalen Unternehmensvision erneut geprüft und weiter konkretisiert. Aufbauend darauf kann die übergeordnete Digitalisierungsstrategie erarbeitet werden, die den Weg zur Zielerreichung beschreibt. Als neues Werkzeug zur Unterstützung dieses "Down-Up"-Vorgehens haben wir eine Methode zur Entwicklung von sogenannten „Digitalen Zielbildern" entwickelt. Unter einem digitalen Zielbild verstehen wir eine prägnante Darstellung des Selbstverständnisses eines Funktionsbereichs im Kontext der Digitalisie- rung auf Basis von vier grundlegenden Bestandteilen (nummeriert in Bild 3).

Die digitale Vision (1) repräsentiert ein konkretes, realisierbares und gleichzeitig distanziertes Zukunftsbild eines Funktionsbereichs im Kontext der Digitalisierung. Sie ist ein wichtiges Führungsinstrument, um eine einheitliche Gesamtorientierung des Funktionsbereichs innerhalb der Digitalisierung zur Geltung zu bringen.

Um die eher abstrakte und langfristig definierte digitale Vision weiter zu konkretisieren, werden digitale Use-Cases (2) entlang der definierten End-to-End-Prozesse des Unternehmens identifiziert. Digitale Use-Cases sind konkrete Digitalisierungsvorhaben, die zur digitalen Transformation eines Funktionsbereichs beitragen (bspw. Einsatz von Predictive Maintenance). Durch die Orientierung an den End-to-EndProzessen erarbeitet jeder Bereich diejenigen digitalen Use-Cases, die zur Verbesserung des Gesamtprozesses beitragen. Zum einen wird somit der Gefahr einer isolierten Optimierung von Einzelabläufen vorgebeugt, zum anderen werden Synergieeffekte zwischen verschiedenen Bereichen identifiziert.

Sechs verschiedene Handlungsdimensionen unterstützen die Einordnung von digitalen Use-Cases zu deren primären Anwendungsbereichen. Die Handlungsdimensionen unter-
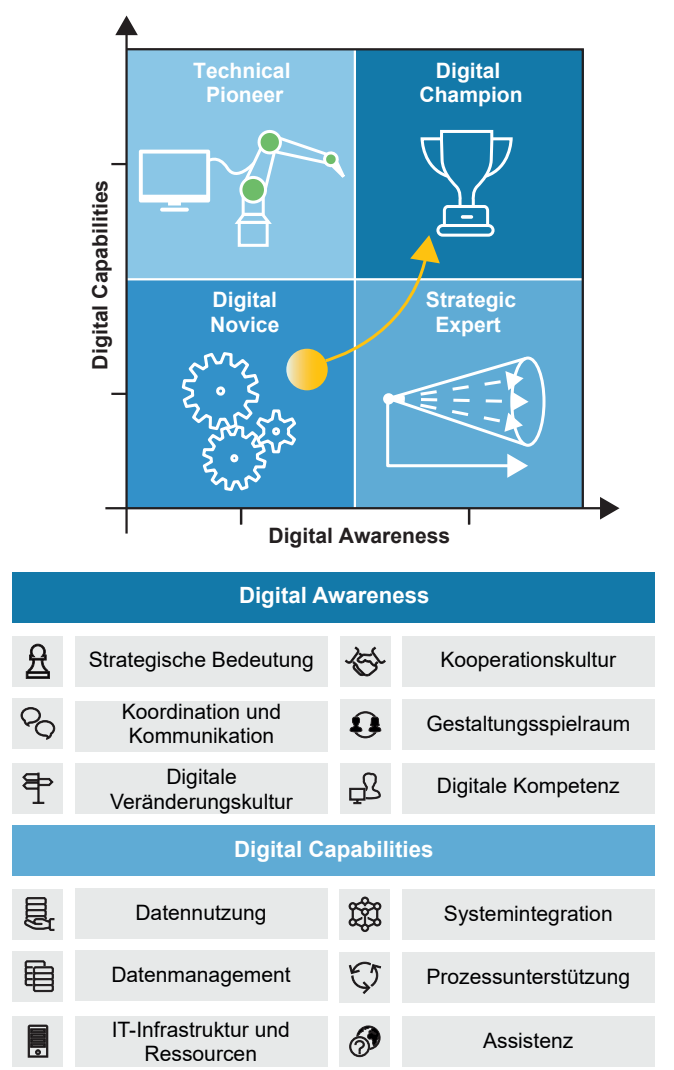

Bild 2: Portfolio und Kriterien zur Reifegradbestimmung. 


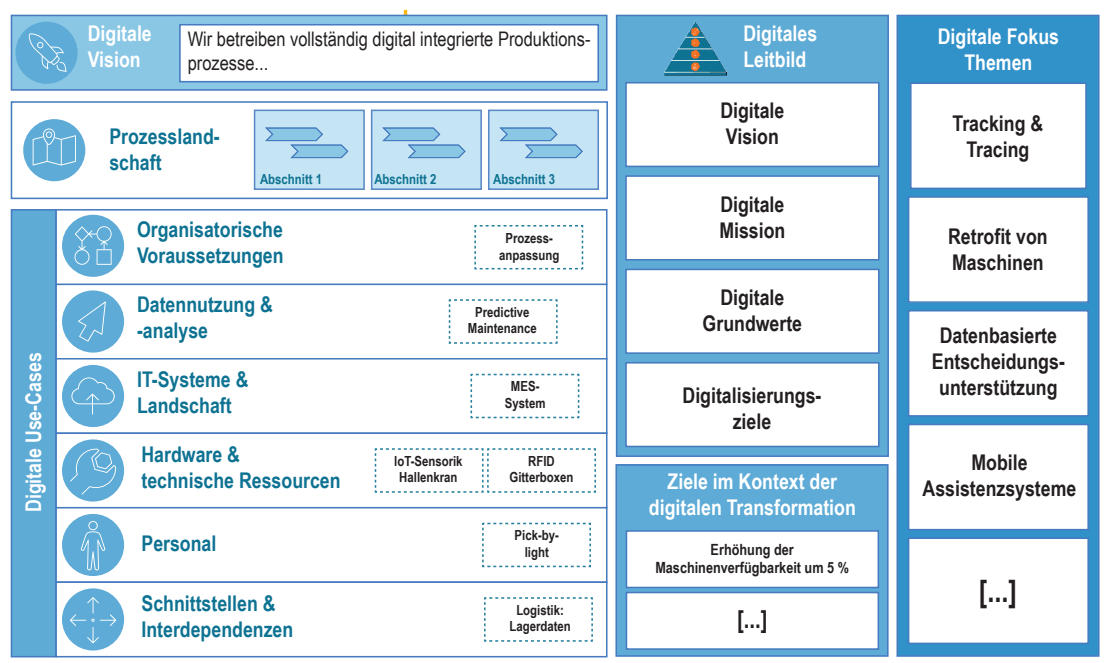

Bild 3: Vorlage zur Erstellung eines digitalen Zielbilds. talen Use-Cases. scheiden dabei organisatorische, technische und personelle Anwendungsbereiche der digi-

Die Erstellung der digitalen Zielbilder sollte jedoch nicht losgelöst dem ersten Entwurf der digitalen Unternehmensvision erfolgen. Vielmehr werden relevante Auszüge der digitalen Unternehmensvision als Leitplanken vorgegeben. Unter Berücksichtigung der digitalen Vision des jeweiligen Funktionsbereichs und der digitalen Use-Cases sind im Rahmen dieser Leitplanken strategischen Ziele im Kontext der Digitalisierung abzuleiten (bspw. die Erhöhung der Maschinenverfügbarkeit durch digitale Technologien) (3).

Die identifizierten digitalen Use-Cases sind die Grundlage zur Ableitung digitaler Fokusthemen (4). Dazu werden digitale Use-Cases mit einer hohen inhaltlichen Nähe zusammengeführt, wodurch eine Identifikation von übergeordneten Themenclustern ermöglicht wird. Diese Themencluster repräsentieren die digitalen Fokusthemen, die innerhalb eines Funktionsbereichs im Kontext der Digitalisierung adressiert werden (bspw. Tracking \& Tracing). Sie dienen der bereichsübergreifenden Kommunikation und Synchronisation der einzelnen Digitalisierungsinitiativen.

Aufbauend auf diese Identifikation von Digitalisierungszielen sowie der zugehörigen digitalen Use-Cases können Unternehmen mit einer Digitalisierungsstrategie den Weg festlegen, wie sie die festgelegten Digitalisierungsziele erreichen können und wollen.

\section{Planung der Umsetzung}

Oftmals identifizieren Unternehmen eine hohe Anzahl an digitalen Use-Cases, die es innerhalb der Umsetzungsplanung zu strukturieren gilt. Wenig erfolgversprechend ist dabei kurzfristiger Aktionismus mit Initiativen ohne nachhaltige Planung. Lohnenswert ist eine objektive, kriterien-basierte Gegenüberstellung, Klassifizierung und Bewertung der identifizierten digitalen Use-Cases. Ein wesentlicher Aspekt der objektiven Bewertung ist dabei die Wirtschaftlichkeitsbetrachtung. Um die identifizierten, digitalen Use-Cases zunächst grundlegend zu strukturieren, nutzen wir ein einfaches, aber praktikables Klassifikationsschema. Dieses fußt auf der Unterscheidung von vier verschiedenen Projektklassen. Mondlandungsprojekte dienen dem Unternehmen dazu, bislang unerschlossene Themen und Technologien über einen mittleren bis langfristigen Zeithorizont zu erforschen. Demgegenüber wird in Leuchtturmprojekten untersucht, ob ein Technologieeinsatz den erhofften Beitrag zur digitalen Transformation leistet und letztlich im gesamten Unternehmen ausgerollt werden kann. Schnellbootprojekte können Betriebe kurzfristig und mit geringen bis mittlerem Implementierungsaufwand und Risiko angehen. Strategische Programme hingegen sind hoch-volumige und langfristige Initiativen mit einem mittleren bis hohen, unternehmerischen Risiko, die zu grundlegenden Änderungen im Unternehmen führen. Aufbauend auf dieser Klassifikation sollten Abhängigkeiten und Synergien zwischen den einzelnen Projekten und Programmen untersucht werden. Eine Bewertung nach unternehmensspezifischen Gesichtspunkten dient als Grundlage für eine Umsetzungsroadmap, die die Projekte in eine strukturierte zeitliche Abfolge bringt.

Die hier skizzierte Vorgehensweise ermöglicht es Unternehmen, ihren individuellen Fahrplan für die digitale Transformation zu erstellen. Ein adäquates Change Management stellt sicher, dass sämtliche Stakeholder kontinuierlich in die grundlegenden Veränderungsprozesse des Unternehmens involviert werden. Beispielhafte Maßnahmen sind regelmäßige Informationsveranstaltungen mit Mitarbeiterinnen und Mitarbeitern, Betriebsräten und Gewerkschaften zu Chancen und Risiken der Digitalisierung. Derartige Aktivitäten sind wichtig, um Bedenken und Vorbehalte gegenüber der Digitalisierung proaktiv zu begegnen und Barrieren abzubauen. Denn nur durch die konsequente Einbindung aller Beteiligten kann die digitale Transformation erfolgreich gestaltet werden.

Schlüsselwörter:

Digitalisierungsstrategie, Digitale Strategie, Digitale Transformation, Digitalisierung, Reifegradmodell, Digitale Zielbilder 